ARTICLE

\title{
Polyoxometalate-based homochiral metal-organic frameworks for tandem asymmetric transformation of cyclic carbonates from olefins
}

Qiuxia Han ${ }^{1,2, \star}$, Bo $\mathrm{Qi}^{1, \star}$, Weimin Ren ${ }^{1}$, Cheng $\mathrm{He}^{1}$, Jingyang $\mathrm{Niu}^{2} \&$ Chunying Duan ${ }^{1,3}$

Currently, great interest is focused on developing auto-tandem catalytic reactions; a substrate is catalytically transferred through mechanistically distinct reactions without altering any reaction conditions. Here by incorporating a pyrrolidine moiety as a chiral organocatalyst and a polyoxometalate as an oxidation catalyst, a powerful approach is devised to achieve a tandem catalyst for the efficient conversion of $\mathrm{CO}_{2}$ into value-added enantiomerically pure cyclic carbonates. The multi-catalytic sites are orderly distributed and spatially matched in the framework. The captured $\mathrm{CO}_{2}$ molecules are synergistically fixed and activated by well-positioned pyrrolidine and amine groups, providing further compatibility with the terminal $\mathrm{W}=\mathrm{O}$ activated epoxidation intermediate and driving the tandem catalytic process in a single workup stage and an asymmetric fashion. The structural simplicity of the building blocks and the use of inexpensive and readily available chemical reagents render this approach highly promising for the development of practical homochiral materials for $\mathrm{CO}_{2}$ conversion.

\footnotetext{
${ }^{1}$ State Key Laboratory of Fine Chemicals, Dalian University of Technology, Dalian 116024, China. ${ }^{2}$ Key Laboratory of Polyoxometalate Chemistry of Henan Province, College of Chemistry and Chemical Engineering, Henan University, Kaifeng 475004, China. ${ }^{3}$ Collaborative Innovation Center of Chemical Science and Engineering, Tianjin 300071, China. ${ }^{\star}$ These authors contributed equally to this work. Correspondence and requests for materials should be addressed to C.D. (email: cyduan@dlut.edu.cn).
} 
$\mathrm{R}$ ecently, the selective and efficient conversion of light olefins with $\mathrm{CO}_{2}$ as a $\mathrm{C}_{1}$ building block into value-added enantiomerically pure cyclic carbonates has attracted great interest from chemists and governments due to its tremendous potential economic and environmental impact, especially considering the increasing worldwide greenhouse effect ${ }^{1}$. The general synthetic procedure includes two mechanistically distinct catalytic processes consisting of the asymmetric epoxidation of olefins, based on the research of Sharpless and others, and the asymmetric coupling epoxide with $\mathrm{CO}_{2}$ (refs 2-5). Both of the catalytic processes require asymmetric (or associated) catalysts to ensure enantioselectivity, as well as a sequential separation and purification process of the products from the reagents, the catalysts and the associated materials. Because light olefins are the most inexpensive and readily available chemical building blocks, a one-pot procedure that processes an olefin through a chain of discrete catalytic steps with multiple transformations using a single workup stage is highly desirable for the environmentally benign conversion of carbon dioxide to fine chemicals ${ }^{6}$.

Inspired by natural enzymatic processes, chemists have attempted to emulate natural reaction cascades by developing auto-tandem catalytic reactions in which a substrate is catalytically transferred through two or more mechanistically distinct reactions without the addition of reactants or catalysts and without altering the reaction conditions ${ }^{7,8}$. Recently, homogeneous organo- and organometallic auto-tandem catalysts have been found to show high efficiency in solution? This feature can be advantageous for separation and catalyst recovery by creating heterogenized catalysts using linkers to attach them to a support, such as high surface-area metal oxide materials, or alternatively, incorporating them within porous metal-organic frameworks (MOFs). One of the key advantageous of MOFs lies in their easily tunable composition in which the constituents' geometry, size and functionality can be widely varied $^{10-12}$. Thus, an ongoing challenge for the development of auto-tandem catalytic processes has been beyond the selection and incorporation of two or more catalytic sites by modifying these moieties in the building block of the original MOFs, but includes the achievement of compatibility between the reaction intermediates and synergy of the multiple catalytic cycles ${ }^{13-15}$.

Polyoxometalates (POMs) are well-known catalysts studied in olefin epoxidation, with excellent thermal and oxidative stability towards oxygen donors ${ }^{16-18}$. By combining chiral organic catalysts and POM components in a single MOF (POMOF), these POMOF materials can initiate the asymmetric dihydroxylation of aryl olefins with excellent stereoselectivity ${ }^{19}$. Because MOFs have exhibited unrivalled efficiency as heterogeneous Lewis acid catalysts for the chemical conversion of $\mathrm{CO}_{2}^{20-23}$, we envision that the combination of chiral organic catalysts, Lewis acid catalysts and oxidation catalysts would be a powerful approach to devise a tandem catalytic process for the chemical transformation of olefins to chiral cyclic carbonate.

By incorporating Keggin-type $\left[\mathrm{ZnW}_{12} \mathrm{O}_{40}\right]^{6-}$ anions, zinc(II) ions, $\mathrm{NH}_{2}$-functionalized bridging links, $\mathrm{NH}_{2}$-bipyridine $\left(\mathrm{NH}_{2}-\mathrm{BPY}\right)$ and asymmetric organocatalytic groups, pyrrolidine-2-yl-imidazole (PYI) within one single MOF, herein we report the design and synthesis of two new enantiomorphs of POMOFs, ZnW-PYI1 and ZnW-PYI2, for the efficient conversion of $\mathrm{CO}_{2}$ into value-added enantiomerically pure cyclic carbonates. Because the intrinsic crystalline properties could provide precise knowledge about the nature and distribution of catalytically active sites and about the potential interactions between the catalytic sites and the adsorbed substrates, the use of POM-based homochiral MOFs offers the potential to facilitate analysis of the intermediates and the catalytic processes of each step of the tandem asymmetric transformation.

\section{Results}

Synthesis and characterization of the POMOFs. The solvothermal reaction of $\mathrm{TBA}_{4} \mathrm{~W}_{10} \mathrm{O}_{32}, \mathrm{Zn}\left(\mathrm{NO}_{3}\right)_{2} \cdot 6 \mathrm{H}_{2} \mathrm{O}$, $\mathrm{NH}_{2}$-BPY and L-N-tert-butoxy-carbonyl-2-(imidazole)-1-pyrrolidine (L-BCIP) in a mixed solvent of acetonitrile and $\mathrm{H}_{2} \mathrm{O}$ gave compound $\mathrm{ZnW}-$ PYI1 in a yield of $68 \%$ (Supplementary Fig. 1). Elemental analyses and powder X-ray diffraction (PXRD) indicated the pure phase of the bulk sample (Supplementary Fig. 2). Circular dichroism spectrum of the bulk sample of $\mathrm{ZnW}-$ PYI1 presented positive cotton effects at $276 \mathrm{~nm}$ $(\theta=146 \mathrm{mdeg})$ and negative cotton effects at $340 \mathrm{~nm}$ $(\theta=-41.2 \mathrm{mdeg})$. The entire spectrum was different from the spectra of the chiral adducts L- BCIP and L- PYI (Supplementary Fig. 3). ZnW-PYI2 was prepared similarly to $\mathrm{ZnW}$ - PYI1, except that D-BCIP replaced L-BCIP. The circular dichroism spectrum of ZnW - PYI2 exhibited the cotton effects that were opposite to ZnW - PYI1, as expected for a pair of enantiomers with a mirror-imager relationship (Fig. 1a). Single-crystal structural analysis revealed that $\mathrm{ZnW}$ - PYI1 and ZnW - PYI2 shared the same cell dimensions crystallized in the chiral space group $P 2_{1}$ (Supplementary Data 1 and 2, Supplementary Fig. 14-21).

The crystal structures of POMOFs. The asymmetric unit of $\mathrm{ZnW}-\mathrm{PYI} 1$ consists of the Keggin anion $\mathrm{ZnW}_{12} \mathrm{O}_{40}{ }^{6-}$ and the cation $\left[\mathrm{Zn}_{2}\left(\mathrm{NH}_{2}-\mathrm{BPY}\right)_{2}(\mathrm{HPYI})_{2}\left(\mathrm{H}_{2} \mathrm{O}\right)\left(\mathrm{CH}_{3} \mathrm{CN}\right)\right]^{6+}$ (Supplementary Fig. 4). The precursor $\mathrm{W}_{10} \mathrm{O}_{32}^{4-}$ anion is labile under hydrothermal conditions and favours to rearrangement into the more stable Keggin-type $\mathrm{ZnW}_{12} \mathrm{O}_{40}{ }^{6-}$ anion. Each of these $\mathrm{ZnW}_{12} \mathrm{O}_{40}{ }^{6-}$ anions connects to four zinc ions through the terminal oxygen atoms and acts as a four connector (Supplementary Fig. 5). Each of the two crystallographically independent zinc ions adopts a distorted octahedral geometry (Supplementary Fig. 6,7) and coordinates to two terminal oxygen atoms from different $\mathrm{ZnW}_{12} \mathrm{O}_{40}{ }^{6-}$ anions, forming a twodimensional square grid sheet (Fig. 1b,c, Supplementary Fig. 8,9). Each of the $\mathrm{NH}_{2}-\mathrm{BPY}$ ligands coordinates to two different zinc ions and further bridges the sheets to form a three-dimensional framework with one-dimensional chiral channels along the $b$ axis (Fig. 1d, Supplementary Fig. 10,11). The accessible empty space of the framework is approximately $289.1 \AA^{3}(8.1 \%)$, as calculated from PLATON analysis, suggesting the possibility that the MOF-based material ZnW - PYI1 can absorb substrates within its pores ${ }^{24}$. Chiral PYI was located in the pores of the MOF, with the butoxycarbonyl of L- BCIP removed simultaneously in the reaction and the pyrrolidine nitrogen was protonated. The unique redox properties of $\mathrm{ZnW}_{12} \mathrm{O}_{40}{ }^{6-}$, with its oxygen-enriched surface, provide sufficient driving force for the transformation of the catalytic precursors to the active intermediate of the epoxidation $^{25,26}$. The chiral PYIs act as cooperative catalytic sites to forge a crucial reaction centre that enhances the activities of the oxidants and drives the catalysis asymmetrically ${ }^{27,28}$. Detailed structural analyses reveal that one zinc ion has a water molecule at its sixth coordination position, while the other is associated with an acetonitrile molecule. The removability of the coordinated water molecules indicates the potential of $\mathrm{ZnW}$ - PYI1 to act as an active Lewis acid catalyst (Supplementary Fig. 12). These amine groups in $\mathrm{NH}_{2}-\mathrm{BPY}$ are positioned around the inner surface of the channels and act as accessible sites to activate $\mathrm{CO}_{2}$ directly, enabling the catalytic performance in the coupling of carbon dioxide with epoxide under relatively mild conditions (Fig. 2) $29-32$.

Separated tandem catalysis. The transformation of asymmetric epoxidation was initially examined using styrene and $t$-butylhydroperoxide (TBHP, $70 \%$ in decane) as the oxidant, along with 
a

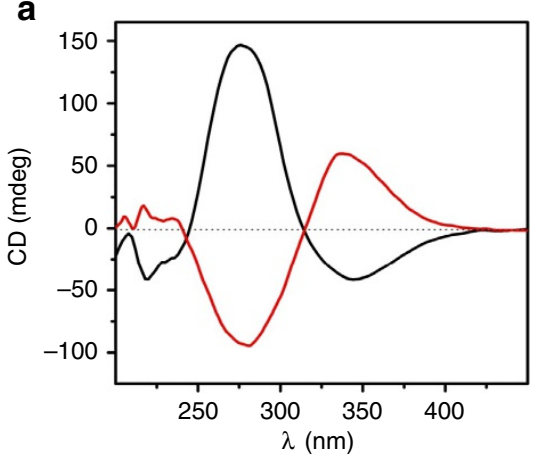

C

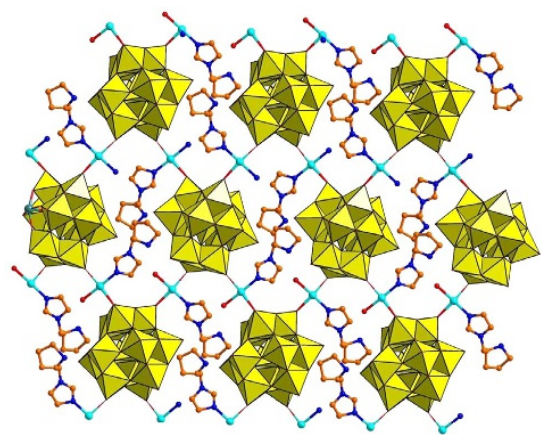

b

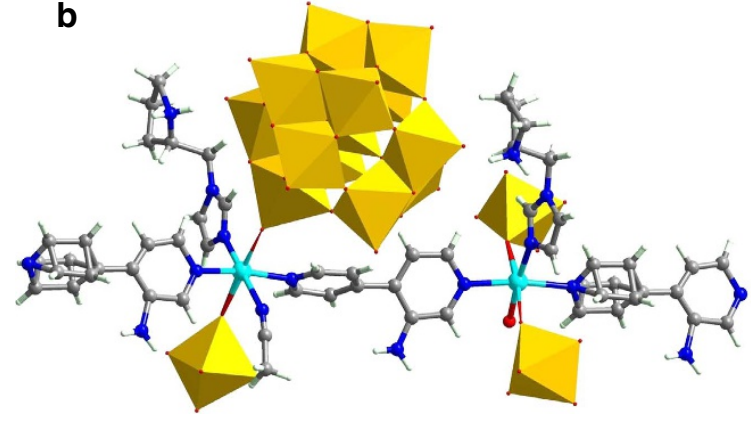

d

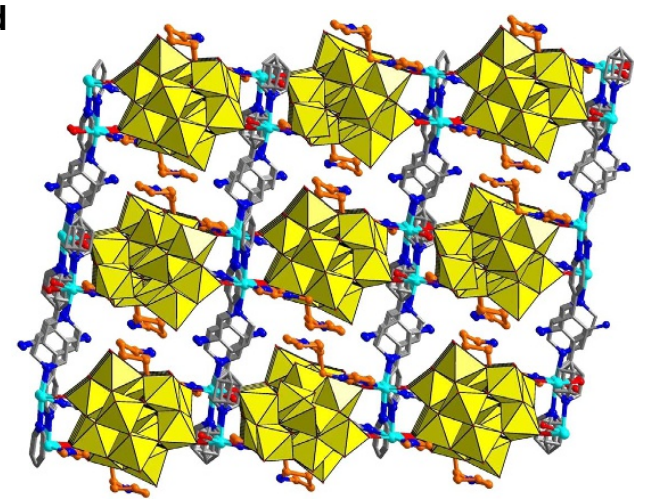

Figure 1 | The structure and characterization of ZnW - PYIs. (a) Circular dichroism spectra of bulk crystals of ZnW - PYI1 (black) and ZnW - PYI2 (red), respectively. (b) Plot of the one-dimensional connections between the bipyridine ligands and the zinc centres, showing the coordination mode of the zinc centres. (c) Perspective view of the two-dimensional sheet connected by the polyoxometalate clusters and the zinc centres, the bipyridine ligands were omitted for clarity. (d) 3D open network of ZnW - PYI1 viewed down the $b$ axis. $\mathrm{H}$ atoms and solvent molecules are omitted for clarity. Carbon, nitrogen and zinc are drawn in gray (orange for PYI), blue, and cyan, respectively, with $\mathrm{ZnW}_{12} \mathrm{O}_{40}{ }^{6-}$ shown as polyhedra.

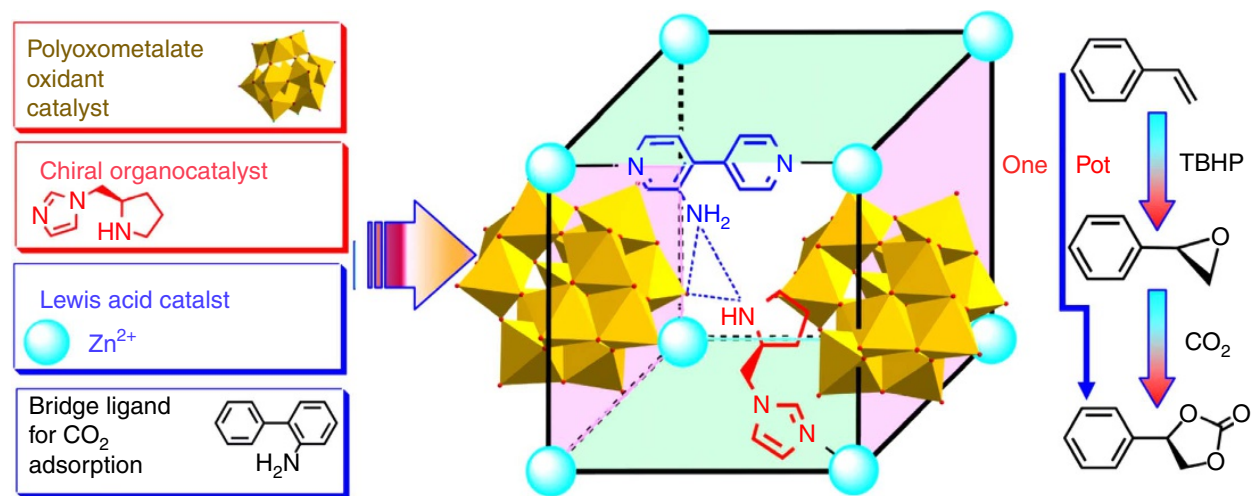

Figure 2 | The design concept of achieving a tandem catalyst. Synthetic procedure of the metal-organic framework, showing the constitutive/ constructional fragments of the MOF; and the schematic representation of tandem catalysis for the asymmetric cyclic carbonate transformation from olefins and carbon dioxide.

$\mathrm{ZnW}-\mathrm{PYI} 1(0.1 \% \mathrm{~mol}$ ratio) in a heterogeneous reaction at $50^{\circ} \mathrm{C}$ for 5 days. As shown in Table 1 (entries 1-4), the result revealed the successful execution of our POMOF design, showing excellent yield $(92 \%)$ and enantioselectivity (79\% enantiomer excess (ee)) for (R)-styrene oxide (Supplementary Fig. 22, Supplementary Table 6). The control experiments showed that L-PYI and its hydrochloride salt could not initiate the reaction under similar conditions. The use of $\mathrm{L}-\mathbf{P Y I}$ and $\mathrm{Zn}_{3}\left[\mathrm{ZnW}_{12} \mathrm{O}_{40}\right]$ as homogeneous catalysts gave a conversion of $55 \%$ and an ee value of $18 \%$. The higher conversion in the case of the $\mathrm{ZnW}$ - PYI1 system was attributed to the suitable distribution of pairs of the chiral PYI moiety and the $\mathrm{ZnW}_{12} \mathrm{O}_{40}{ }^{6-}$ oxidant catalyst. Infrared of the catalyst impregnated with TBHP revealed a new $v(\mathrm{O}-\mathrm{O})$ band at $821 \mathrm{~cm}^{-1}$, which indicated that the active intermediate peroxotungstate was formed during the epoxidation process (Fig. 3a) ${ }^{33}$. From a mechanistic perspective, the formation of hydrogen bonds between the pyrrolidine $\mathrm{N}$ atom and the terminal oxygen atoms of the $\mathrm{ZnW}_{12} \mathrm{O}_{40}{ }^{6-}$ with the interatomic separation $\mathrm{N}(12) \cdots \mathrm{O}(7)$, approximately $2.8 \AA$, initially activated the corresponding $\mathrm{W}=\mathrm{O}_{\mathrm{t}}\left(\mathrm{O}_{\mathrm{t}}=\right.$ terminal oxygen $)$ and generated an active peroxide tungstate intermediate, ensuring the smooth progress of the reaction (Supplementary Table 2-5). These hydrogen bonds then enforced the proximity between the conventional electrophilic oxidant and the chiral directors to 
Table 1 | Yields and enantiomer excess (ee) in the asymmetric epoxidation of the olefins ${ }^{\star}$, in the coupling of $\mathrm{CO}_{2}$ to styrene oxide $^{\dagger}$, and in the asymmetric auto-tandem catalysis of olefins to cyclic carbonates over $\mathbf{Z n W}$ - PYI catalysts ${ }^{\ddagger}$.

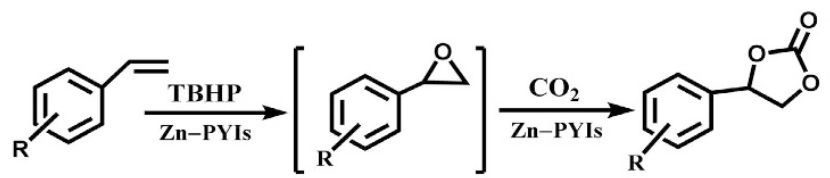

Entry

*Entries 1-4: Conditions: olefin: $10 \mathrm{mmol}, \mathrm{ZnW}$ - PYls: $0.01 \mathrm{mmol}$; TBHP(70\% in decane): $20 \mathrm{mmol}, 50^{\circ} \mathrm{C}, 120 \mathrm{~h}$.

tEntries 5-8: Conditions: styrene oxide: $10 \mathrm{~mol}$, catalyst: $0.01 \mathrm{mmol}, \mathrm{TBABr} 0.1 \mathrm{mmol}, \mathrm{CO}_{2}, 0.5 \mathrm{MPa}, 50^{\circ} \mathrm{C}, 48 \mathrm{~h}$.

FEntries 9-12: conditions: olefin: $10 \mathrm{mmol}$, catalyst: $0.01 \mathrm{mmol}$, TBHP $20 \mathrm{mmol}, \mathrm{CO}_{2}, 0.5 \mathrm{MPa}, 50^{\circ} \mathrm{C}, 96 \mathrm{~h}$

\$The yield was determined by ${ }^{1} \mathrm{H}$ NMR spectroscopy of crude products. Yields catalysed by $\mathrm{ZnW}-\mathbf{P Y I} 2$ were listed in the parentheses.

IIThe ee value was determined by chiral HPLC on a Chiralcel OD - H column. The ee values catalysed by ZnW - PYI2 were listed in the parentheses.

provide additional steric orientation, driving the catalysis to proceed in a stereoselective manner ${ }^{19,34}$.

Solids of ZnW - PYI2 exhibited similar catalytic activities but gave products with the opposite chirality in the asymmetric epoxidation of styrene. The use of such a catalyst can be extended to other substituted styrene substrates and $\alpha, \beta$-unsaturated aldehydes with comparable activity and asymmetric selectivity. The presence of a $\mathrm{C}=\mathrm{O}$ stretching vibration at $1,675 \mathrm{~cm}^{-1}$ (cf. $1682 \mathrm{~cm}^{-1}$ of the free aldehyde) in the infrared spectra of the catalyst impregnated with a $\mathrm{CH}_{2} \mathrm{Cl}_{2}$ solution of cinnamaldehyde suggested the absorbance and activation of cinnamaldehyde in the cavities of ZnW - PYI1 (Fig. 3b).

The transformation of the asymmetric coupling of $\mathrm{CO}_{2}$ to styrene oxide was examined using the racemic styrene oxide and $\mathrm{CO}_{2}$ in free solvent, along with $\mathrm{ZnW}-$ PYI1 (0.1\% mol ratio) and cocatalyst $\mathrm{TBABr}(1 \% \mathrm{~mol}$ ratio), in a heterogeneous mixture at $50^{\circ} \mathrm{C}$ and $0.5 \mathrm{MPa}$ for $48 \mathrm{~h}$, as shown in Table 1 (entry 5). The result exhibited excellent reaction efficiency ( $>99 \%$ yield) for phenyl(ethylene carbonate) (Supplementary Table 7). The control experiments demonstrated that no detectable conversion was observed for the model reaction in the absence of $\mathrm{ZnW}-$ PYI1 or the ammonium salt cocatalyst. It is postulated that the directed coordination of the $\mathrm{ZnW}_{12} \mathrm{O}_{40}{ }^{6-}$ anion to the zinc ions results in significant activation of the MOF on the acid surface, facilitating the coupling step because of its additional labile ligand sites, possibly through the provision of a carbon dioxide. The surface properties of the nanocation also meet a number of criteria for the carbon dioxide coupling reaction, such as an overall positive charge, the presence of coordinated water and the presence of functional groups for acid catalysis. 
a

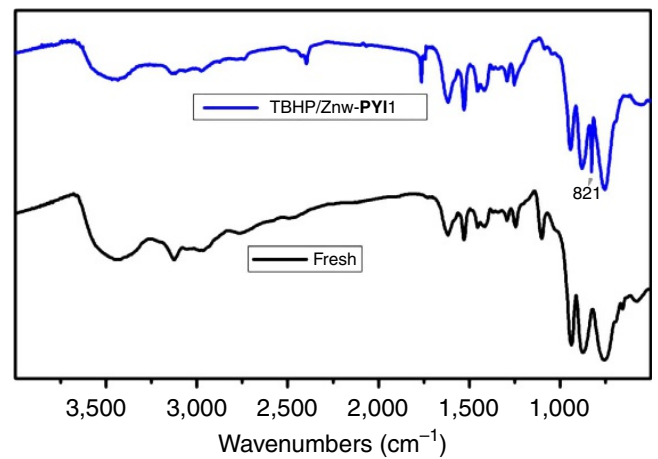

b

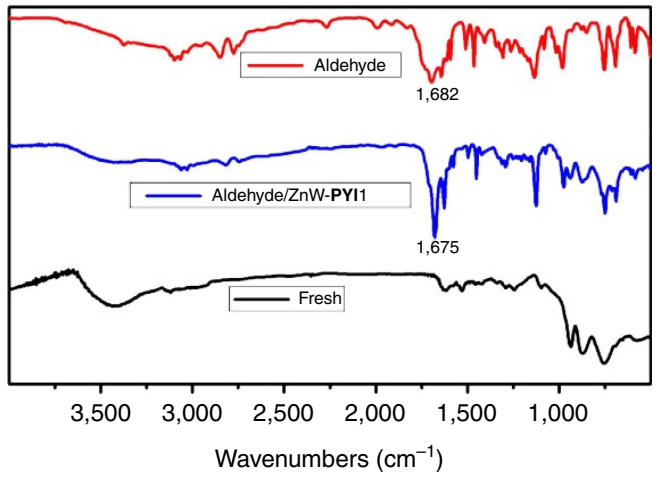

C

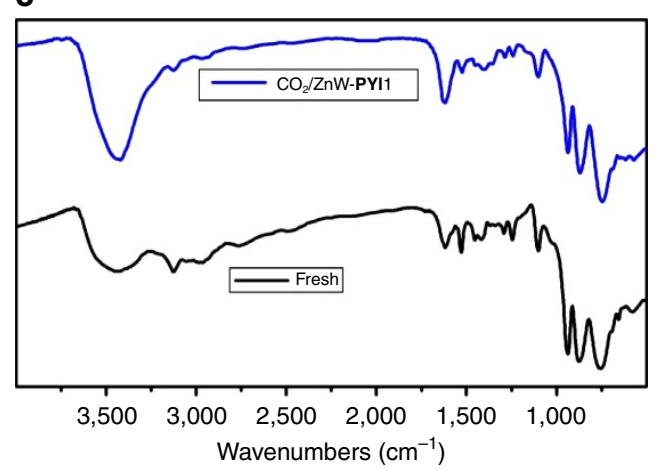

d

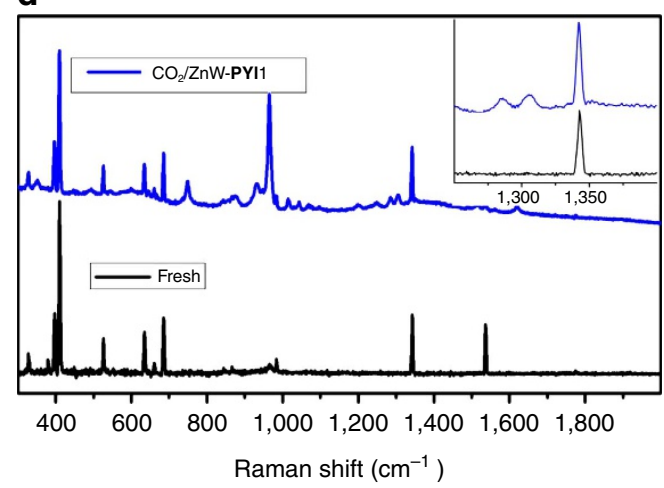

Figure 3 | The spectroscopic studies of ZnW - PYI1. (a) Infrared (IR) spectra of fresh and TBHP-oxidized ZnW - PYI1. (b) IR spectra of fresh and cinnamaldehyde-incorporated ZnW - PYI1. (c,d) IR spectra and Raman spectra of fresh (dark line) and $\mathrm{CO}_{2}$-adsorbed $\mathrm{ZnW}$ - PYI1 (blue line), respectively.

Enantiospecific phenyl(ethylene carbonate) could be obtained through the coupling of $\mathrm{CO}_{2}$ to the $(R)$ or $(S)$-styrene oxide, catalysed with excellent reaction efficiency ( $>99 \%$ yield) and high enantioselectivity ( $>90 \%$ ee) (Supplementary Fig. 23, Supplementary Table 8) by ZnW-PYIs (Table 1, entries 6,7). The retention of chirality through the asymmetric coupling process demonstrated that selective ring opening occurred preferentially at the methylene $\mathrm{C}-\mathrm{O}$ bond of the terminal epoxides 5,32 . The high selectivity of the asymmetric transformation reflected that $\mathrm{CO}_{2}$ molecules were also activated, providing favourable conditions for a fast reaction with epoxide by nucleophilic attack, avoiding the racemization of the epoxide through premature ring opening. It is postulated that these $\mathrm{NH}_{2}$ molecules in channels not only enhanced the reaction rate by increasing the concentration of $\mathrm{CO}_{2}$ substrate around its reactive centre but also increased the electron cloud density of activated $\mathrm{CO}_{2}$, enabling the cyclic carbonate ring formation ${ }^{35}$. In contrast to Ahn's group reported that combination of Lewis acid and base give highly active for the $\mathrm{CO}_{2}$ cycloaddition to epoxide because of cooperative activation of epoxide by acid-base ${ }^{22}$, the Lewis acid catalytic zinc centres restricted within the $\mathrm{ZnW}$-PYIs channels have the potential to interact synergistically with $\mathrm{CO}_{2}$ in view of spatial location matching of catalytic sites in MOFs, such that an intramolecularly cooperative catalysis is proposed to contribute to the high activity and excellent stereochemical control of the given reactions ${ }^{36}$.

The irreversible $\mathrm{CO}_{2}$-adsorption and desorption isotherms of ZnW - PYI1 may be attributed to the chemical adsorption onto the amine of $\mathrm{NH}_{2}$-BPY (Supplementary Fig. 13) (ref. 37). The infrared spectrum of activated $\mathrm{ZnW}-$ PYI1 in a vacuum and after the introduction of 1 bar of $\mathrm{CO}_{2}$ at room temperature clearly shows the loss of the $\mathrm{NH}$ stretching vibration peak at $3,126 \mathrm{~cm}^{-1}$, confirming that the $\mathrm{CO}_{2}$ was adsorbed and activated by $\mathrm{NH}_{2}$ groups in the channels of $\mathrm{ZnW}-$ PYI1 (Fig. 3c) (refs 38,39). The Raman spectra of the MOF in a vacuum and after the introduction of 1 bar of $\mathrm{CO}_{2}$ showed peaks at $1,285 \mathrm{~cm}^{-1}$ (ref. 40), further supporting that $\mathrm{CO}_{2}$ molecules were adsorbed within the MOFs (Fig. 3d). Mechanistically, this reaction is based on a nucleophilic cocatalyst that activates the epoxide to form an alkoxide. This intermediate can then react with activated carbon dioxide to ultimately yield the cyclic carbonate.

One-pot asymmetric catalysis. Because chiral $\mathrm{ZnW}-$ PYIs demonstrated their ability as effective catalysts in the epoxidation of styrene and the coupling reaction, the one-pot synthesis was applicable to a range of different substrates. As shown in Table 1 (entries 9-11), by heating a reaction mixture of styrene, TBHP and $\mathrm{CO}_{2}$ with catalyst $\mathrm{ZnW}-\mathrm{PYI} 1$ to $50^{\circ} \mathrm{C}$, the asymmetric epoxidation and the $\mathrm{CO}_{2}$ asymmetric coupling could be smoothly completed with a single workup stage. The target $(R)$-phenyl(ethylene carbonate) was obtained in $92 \%$ yield with $80 \%$ ee (Supplementary Fig. 24, Supplementary Table 9). The removal of $\mathrm{ZnW}$ - PYI1 by filtration after $48 \mathrm{~h}$ stopped the reaction, and the filtrate afforded nearly no additional conversion after stirring for another $48 \mathrm{~h}$. These observations suggest that $\mathrm{ZnW}-$ PYI1 is a true heterogeneous catalyst. $\mathrm{ZnW}$ - PYI1 solids could be isolated from the reaction suspension by simple filtration. The catalysts could be reused at least three times with moderate loss of activity (from 92 to $88 \%$ yield) and with a slight decrease in selectivity (from 80 to $77 \%$ ee) (Supplementary Table 10). The index of PXRD patterns of the $\mathrm{ZnW}-$ PYI1 bulky sample filtered from the catalytic reaction revealed that the crystallinity was maintained. The ZnW - PYI2 solids exhibited similar catalytic activities but 
a

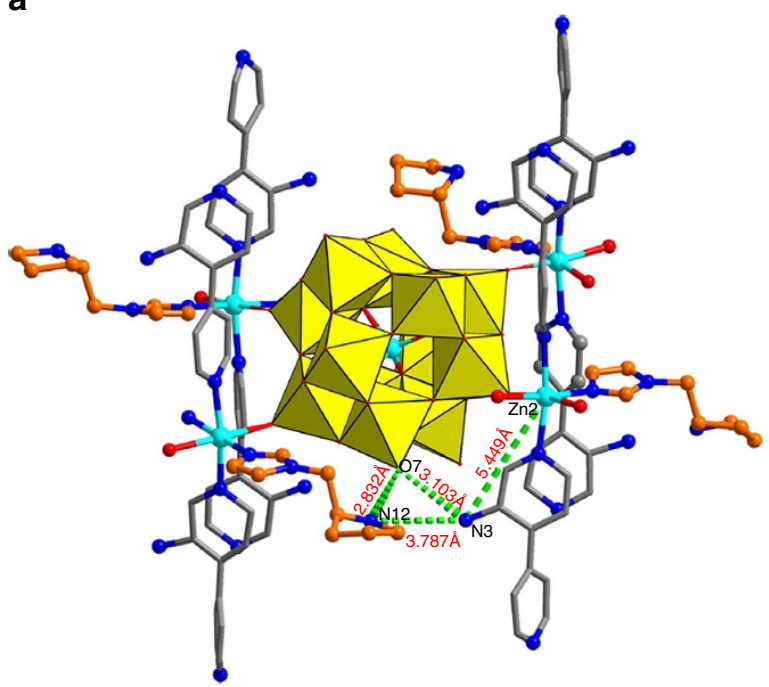

b

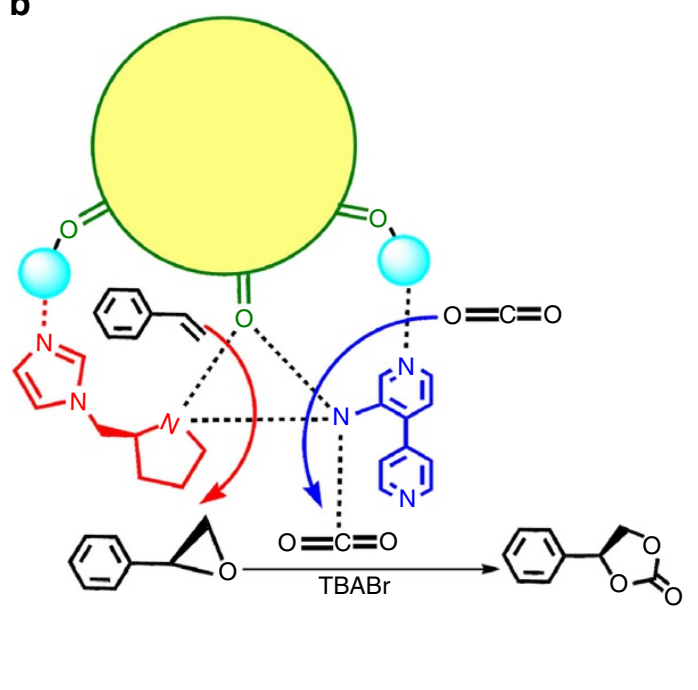

Figure 4 | The spatial relationship of multiple catalytic sites and synergetic catalysis. (a) Schematic diagram of the catalytic site distribution in $\mathrm{ZnW}$ - PYI1, showing the interactions of the catalytic sites. (b) Diagram of potential mechanism for the auto-tandem catalysis.

gave products with opposite chiralities in the asymmetric autotandem epoxidation/coupling of styrene.

\section{Discussion}

It was found that performing the reaction through a one-pot process could greatly shorten the reaction time to 4 days, and the configuration was maintained throughout both of the steps. It is clear from the crystal structures of the $\mathrm{ZnW}$ - PYIs that the smooth and stereoselective conversion of the olefins into the epoxide can be attributed to the hydrogen bonding interaction between the spatially matched organocatalyst PYI and the oxidation catalyst $\mathrm{ZnW}_{12} \mathrm{O}_{40}{ }^{6-}$ and to the potential $\pi-\pi$ interaction between the benzene ring of styrene oxide and the imidazole ring of PYI. Because the $\mathrm{CO}_{2}$ molecules adsorbed in the channels of $\mathrm{ZnW}-\mathbf{P Y I} 1$ are activated by $\mathrm{NH}_{2}$ groups, the weak hydrogen bonding interactions between the chiral amine group $\mathrm{N}(12)$ and the amino group $\mathrm{N}(3)$ (interatomic separation of $3.79 \AA$ ) is believed to enforce the proximity between the activated $\mathrm{CO}_{2}$ and the terminal epoxide (Fig. 4). The well-matched positions and the suitable interactions provide a promising route for nucleophilic attack at the methylene $\mathrm{C}-\mathrm{O}$ bond, ensuring chiral retention in the coupling process. The Lewis acid catalytic zinc centres restricted within the MOF channels have the potential to interact synergistically with $\mathrm{CO}_{2}$ such that the compatibility between the reaction intermediates and the synergy of the multiple catalytic cycles allow the auto-tandem reaction to proceed smoothly and efficiently ${ }^{36}$. Most importantly, the multi-catalytic sites with orderly distribution and spatial matching in the three-dimensional open framework provide favourable conditions for the auto-tandem reaction and avoid cross-interference.

The use of this catalyst can be extended to other styrene derivatives with comparable activity and asymmetric selectivity. In contrast to the smooth reaction of substrates $9-11$, the one-pot catalytic reaction in the presence of cinnamaldehyde only gave $25 \%$ conversion under the same reaction conditions (Table 1, entry 12). It is suggested that the aldehyde group has the potential to interact with the $\mathrm{NH}_{2}$ group, inactivating these sites for the activation and concentration of $\mathrm{CO}_{2}$, which further substantiates the pivotal role of $\mathrm{NH}_{2}$ in the one-pot process.
We have developed an asymmetric auto-tandem epoxidation/ cycloaddition catalytic reaction catalysed solely by chiral POMOFs, which proceeds in a highly enantioselective manner for the efficient conversion of light olefins into value-added enantiomerically pure cyclic carbonates in a one-pot procedure. The results demonstrated that asymmetric auto-tandem catalysis is an atom-economical and environmentally benign synthetic method for producing useful chiral compounds.

Future work will focus on design structurally diverse chiral POMOFs and optimizing such reactions. The structural simplicity of catalyst and the use of inexpensive and readily available chemical reagents render this approach highly promising for the development of practical homochiral materials for asymmetric catalytic reactions.

\section{Methods}

Reagents and Syntheses. All chemicals were of reagent grade quality obtained from commercial sources and used without further purification. All epoxides were purchased from Acros and distilled under a nitrogen atmosphere from $\mathrm{CaH}_{2}$ prior to use. Carbon dioxide (99.995\%) was purchased from Dalian Institute of Special Gases and used as received. All manipulations involving air- and/or water-sensitive compounds were carried out in glove box or under dry nitrogen using standard Schlenk techniques. L- and D-N-tert-butoxycarbonyl-2-(imidazole)-1-pyrrolidine ( $\mathrm{L}-$ or D- BCIP) (ref. 41), 3-amino-4,4' -bipyridine $\left(\mathrm{NH}_{2}\right.$-BPY) (ref. 42$)$, and $\left[\left(\mathrm{n}^{-} \mathrm{C}_{4} \mathrm{H}_{9}\right)_{4} \mathrm{~N}\right]_{4}$ $\left[\mathrm{W}_{10} \mathrm{O}_{32}\right]$ (ref. 43) were prepared according to the literature methods.

Elemental analyses. Elemental analyses of $\mathrm{C}, \mathrm{H}$ and $\mathrm{N}$ were performed on a Vario EL III elemental analyser.

Inductively coupled plasma. $\mathrm{W}$ and $\mathrm{Zn}$ analyses were performed on a JarrelAshJ-A1100 spectrometer.

Fourier translation infrared spectrum (FT-IR). FT-IR spectra were recorded as $\mathrm{KBr}$ pelletson JASCO FT/IR-430.

Powder X-ray diffractograms. PXRDs were obtained on a Riguku D/Max-2,400 X-ray diffractometer with $\mathrm{Cu}$ sealed tube $(\lambda=1.54178 \AA)$.

Circular dichroism spectrum. Circular dichroism spectra were measured on JASCO J-810 with solid KBr tabletting.

Laser-Raman spectrum. Raman spectroscopy (Lab Raman HR Evolution) measurements were performed using a solid state $785 \mathrm{~nm}$ laser. A laser power of 
$1-2.5 \%$ was used to avoid degradation of the sample under the laser beam during the Raman measurements.

Thermogravimetric analysis. Thermogravimetric analyses were performed on a Mettler-Toledo TGA/SDTA851 instrument and recorded under $\mathrm{N}_{2}$ or under air, upon 14 equilibration at $100^{\circ} \mathrm{C}$, followed by a ramp of $5^{\circ} \mathrm{C} \mathrm{min}-1$ up to $800^{\circ} \mathrm{C}$.

Gas adsorption isotherms. Gas adsorption isotherms were collected using a Micromeritics 3 Hex 128 instrument. As-synthesized crystals were thoroughly washed with anhydrous dichloromethane and dried under argon flow, approximately $100 \mathrm{mg}$ of each sample was added into a pre-weighed sample analysis tube. The samples were degassed at $100^{\circ} \mathrm{C}$ under vacuum for $24-48 \mathrm{~h}$ until the pressure change rate was no more than $3.5 \mathrm{mTorr} \mathrm{min}^{-1}$. Ultra high purity (UHP) grade $\mathrm{N}_{2}$ and $\mathrm{CO}_{2}$ gas adsorbates $(99.999 \%)$ were used in this study.

Nuclear magnetic resonance. ${ }^{1} \mathrm{H}$ and ${ }^{13} \mathrm{C}$ nuclear magnetic resonance (NMR) spectra were recorded on a Varian INOVA-400 MHz type $\left({ }^{1} \mathrm{H}, 400 \mathrm{MHz} ;{ }^{13} \mathrm{C}\right.$, $400 \mathrm{MHz}$ ) spectrometer. Their peak frequencies were referenced versus an internal standard (tetramethylsilane (TMS)) shifts at 0 p.p.m. for ${ }^{1} \mathrm{H}$ NMR and against the solvent, chloroform-D at 77.0 p.p.m. for ${ }^{13} \mathrm{C}$ NMR, respectively.

High-performance liquid chromatography. High-performance liquid chromatography analysis was performed on Agilent 1,150 using a ChiralPAk OD - H column or $\mathrm{AD}-\mathrm{H}$ column purchased from Daicel Chemical Industries, Ltd.

Crystallography. Data of POMOFs ZnW - PYIs were collected on a Bruker SMART APEX CCD diffractometer with graphite-monochromated Mo-K $\alpha(\lambda=0.71073 \AA)$ using the SMART and SAINT programs ${ }^{44,45}$. Routine Lorentz polarization and Multi-scan absorption correction were applied to intensity data. Their structures were determined and the heavy atoms were found by direct methods using the SHELXTL-97 program package ${ }^{46}$. The remaining atoms were found from successive full-matrix least-squares refinements on $F^{2}$ and Fourier syntheses. Not all the non-hydrogen atoms were refined anisotropically. Hydrogen atoms within the ligand backbones were fixed geometrically at their positions and allowed to ride on the parent atoms. Crystallographic data for $\mathrm{ZnW}$ - PYIs are summarized in Supplementary Table 1.

Synthesis of $\mathbf{Z n W}$ - PYI1. A mixture of $\left[\left(\mathrm{n}-\mathrm{C}_{4} \mathrm{H}_{9}\right)_{4} \mathrm{~N}\right]_{4}\left[\mathrm{~W}_{10} \mathrm{O}_{32}\right](66.4 \mathrm{mg}$, $0.02 \mathrm{mmol}), \mathrm{Zn}\left(\mathrm{NO}_{3}\right)_{2} \cdot 6 \mathrm{H}_{2} \mathrm{O}(60.0 \mathrm{mg}, 0.20 \mathrm{mmol}), \mathrm{NH}_{2}-\mathrm{BPY}(10.3 \mathrm{mg}$, $0.06 \mathrm{mmol})$ and $\mathrm{L}-\mathbf{B C I P}(7.5 \mathrm{mg}, 0.03 \mathrm{mmol})$ in mixed water $(4.0 \mathrm{ml})$ and acetonitrile $(2.0 \mathrm{ml})$ was stirred and its $\mathrm{pH}$ value was adjusted to 4.3 with $1 \mathrm{moll}^{-1}$ acetic acid (HAc). The resulting suspension was sealed in a $25 \mathrm{ml}$ Teflon-lined reactor and kept at $130{ }^{\circ} \mathrm{C}$ for 3 days. After cooling the autoclave to room temperature, yellow rod-like single crystals were separated, washed with water and air-dried. (Yield: calculated (calcd) $68 \%$ based on $\left[\left(\mathrm{n}-\mathrm{C}_{4} \mathrm{H}_{9}\right)_{4} \mathrm{~N}\right]_{4}\left[\mathrm{~W}_{10} \mathrm{O}_{32}\right]$ ). Elemental analyses and inductively coupled plasma calcd (\%) for $\mathrm{C}_{40} \mathrm{H}_{54} \mathrm{~N}_{14} \mathrm{O}_{41} \mathrm{~W}_{12} \mathrm{Zn}_{3}$ : C 12.68, H 1.44, N 5.18, Zn5.18, W 58.22; Found: C 12.64, H 1.41, N 5.20, Zn5.22, W 58.24 for ZnW - PYI1. IR (KBr): 3,440 (s), 3,123(w), $1,619(\mathrm{~s}), 1,532(\mathrm{~s}), 1,247(\mathrm{~s}), 1,103(\mathrm{w}), 938(\mathrm{~s}), 872(\mathrm{~s}), 756$ (versus) per $\mathrm{cm}$.

Synthesis of ZnW - PYI2. The preparation of ZnW - PYI2 was similar to that of $\mathrm{ZnW}$ - PYI1, except that D- BCIP $(50.0 \mathrm{mg}, 0.2 \mathrm{mmol})$ replaced $\mathrm{L}-\mathbf{B C I P}$. (Yield: $c a$. $68 \%$ based on $\left.\left[\left(\mathrm{n}-\mathrm{C}_{4} \mathrm{H}_{9}\right)_{4} \mathrm{~N}\right]_{4}\left[\mathrm{~W}_{10} \mathrm{O}_{32}\right]\right)$. Elemental analyses and inductively coupled plasma calcd (\%) for $\mathrm{C}_{40} \mathrm{H}_{54} \mathrm{~N}_{14} \mathrm{O}_{41} \mathrm{~W}_{12} \mathrm{Zn}_{3}$ : C $12.68, \mathrm{H}$ 1.44, N 5.18, Zn5.18, W 58.22; Found: C 12.64, H 1.42, N 5.17, Zn 5.20, W 58.25 for ZnW - PYI2. IR (KBr): 3,443 (s), 3,124(w), 1,618(s), 1,531(s), 1,248 (s), 1,104(w), 939(s), 873(s), 757(versus) per $\mathrm{cm}$.

Typical one-pot procedure for asymmetric catalysis. Catalyst $(0.01 \mathrm{mmol})$, TBABr $(0.1 \mathrm{mmol})$, TBHP $(20 \mathrm{mmol})$ and styrene $(10 \mathrm{mmol})$ was added to a Schlenk flask $(50 \mathrm{ml})$ equipped with a three-way stopcock. Then $\mathrm{CO}_{2}$ was charged into the autoclave, and the pressure $(0.5 \mathrm{MPa})$ was kept constant during the reaction. The autoclave was put into a bath and heated to the $50{ }^{\circ} \mathrm{C}$. After the expiration of the desired time, the excess gases were vented. The remaining mixture was degassed and fractionally distilled under reduced pressure or purified by column chromatography on silica gel to obtain the cyclic carbonate.

\section{References}

1. Raman, S. K., Brulé, E., Tschan, M. J. L. \& Thomas, C. M. Tandem catalysis: a new approach to polypeptides and cyclic carbonates. Chem. Commun. 50, 13773-13776 (2014).

2. Zhu, Y. G., Wang, Q., Cornwall, R. G. \& Shi, Y. A. Metal-catalyzed asymmetric sulfoxidation, epoxidation and hydroxylation by hydrogen peroxide. Chem. Rev. 114, 8199-8256 (2014).
3. Katsuki, T. \& Sharpless, K. B. The first practical method for asymmetric epoxidation. J. Am. Chem. Soc. 102, 5974-5976 (1980).

4. Zhang, S. L., Huang, Y. Z., Jing, H. W., Yao, W. X. \& Yan, P. Chiral ionic liquids improved the asymmetric cycloaddition of $\mathrm{CO}_{2}$ to epoxides. Green Chem. 11, 935-938 (2009).

5. Ren, W. M., Liu, Y. \& Lu, X. B. Bifunctional Aluminum Catalyst for $\mathrm{CO}_{2}$ Fixation: Regioselective ring opening of three-membered heterocyclic compounds. J. Org. Chem. 79, 9771-9777 (2014).

6. Sun, J. S. et al. Direct synthetic processes for cyclic carbonates from olefins and $\mathrm{CO}_{2}$. Catal. Surv. Asia 15, 49-54 (2011).

7. Abou-Shehada, S. \& Williams, J. M. Separated tandem catalysis, It's about time.. Nat. Chem. 6, 12-13 (2014).

8. Li, L. \& Herzon, S. B. Temporal separation of catalytic activities allows antiMarkovnikov reductive functionalization of terminal alkynes. Nat. Chem. 6, 22-27 (2014).

9. Grondal, C., Jeanty, M. \& Enders, D. Organocatalytic cascade reactions as a new tool in total synthesis. Nat. Chem. 2, 167-178 (2010).

10. Yoon, M., Srirambalaji, R. \& Kim, K. Homochiral metal-organic frameworks for asymmetric heterogeneous catalysis. Chem. Rev. 112, 1196-1231 (2012).

11. Cook, T. R., Zheng, Y. R. \& Stang, P. J. Metal - organic frameworks and selfassembled supramolecular coordination complexes: comparing and contrasting the design, synthesis, and functionality of metal - organic materials. Chem. Rev. 113, 734-777 (2013).

12. An, J. et al. Metal-adeninate vertices for the construction of an exceptionally porous metal-organic framework. Nat. Commun 3, 604 (2012).

13. Bromberg, L., Su, X. \& Hatton, T. A. Heteropolyacid-functionalized aluminum 2-amino-terephtha-late metal-organic frameworks as reactive aldehyde sorbents and catalysts. ACS Appl. Mater. Interfaces 5, 5468-5477 (2013).

14. Zhang, Z. M. et al. Photosensitizing metal - organic framework enabling visible-light driven proton reduction by a Wells - Dawson-type polyoxometalate. J. Am. Chem. Soc. 137, 3197-3200 (2015).

15. $\mathrm{Wu}, \mathrm{P}$. Y. et al. Photoactive chiral metal-organic frameworks for light-driven asymmetric $\alpha$-alkylation of aldehydes. J. Am. Chem. Soc. 134, 14991-14999 (2012).

16. Mizuno, N. \& Kamata, K. Catalytic oxidation of hydrocarbons with hydrogen peroxide by vanadium-based polyoxometalates. Coord. Chem. Rev. 255, 2358-2370 (2011).

17. Du, D. Y., Qin, J. S., Li, S. L., Su, Z. M. \& Lan, Y. Q. Recent advances in porous polyoxometalatebased metal-organic framework materials. Chem. Soc. Rev. 43 4615-4632 (2014).

18. Hua, Z. K., Fu, X. K., Li, Y. D. \& Tu, X. B. Highly efficient and excellent reusable catalysts of molybdenum(VI) complexes grafted on ZPS-PVPA for epoxidation of olefins with tert-BuOOH. Appl. Organometal. Chem. 25, 128-132 (2011)

19. Han, Q. X. et al. Engineering chiral polyoxometalate hybrid metal - organic frameworks for asymmetric dihydroxylation of olefins. J. Am. Chem. Soc. 135, 10186-10189 (2013).

20. Kim, J., Kim, S. N., Jang, H. G., Seo, G. \& Ahn, W. S. $\mathrm{CO}_{2}$ cycloaddition of styrene oxide over MOF catalysts. Appl. Catal. A Gen. 453, 175-180 (2013).

21. Feng, D. W. et al. Construction of ultrastable porphyrin $\mathrm{Zr}$ metal - organic frameworks through linker elimination. J. Am. Chem. Soc. 135, 17105-17110 (2013).

22. Gao, W. Y. et al. Crystal engineering of an nbo topology metal-organic framework for chemical fixation of $\mathrm{CO}_{2}$ under ambient conditions. Angew. Chem. Int. Ed. 53, 2615-2619 (2014).

23. Beyzavi, M. H. et al. A Hafnium-based metal-organic framework as an efficient and multifunctional catalyst for facile $\mathrm{CO}_{2}$ fixation and regioselective and enantioretentive epoxide activation. J. Am. Chem. Soc. 136, 15861-15864 (2014).

24. Spek, A. L. Single-crystal structure validation with the program PLATON. J. Appl. Crystallogr. 36, 7-13 (2003).

25. Mizuno, N., Yamaguchi, K. \& Kamata, K. Epoxidation of olefins with hydrogen peroxide catalyzed by polyoxometalates. Coord. Chem. Rev. 249, 1944-1956 (2005).

26. Duncan, D. C., Chambers, R. C., Hecht, E. \& Hill, C. L. Mechanism and dynamics in the $\mathrm{H}_{3}\left[\mathrm{PW}_{12} \mathrm{O}_{40}\right]$ catalyzed selective epoxidation of terminal olefins by $\mathrm{H}_{2} \mathrm{O}_{2}$. Formation, reactivity, and stability of $\left\{\mathrm{PO}_{4}\left[\mathrm{WO}\left(\mathrm{O}_{2}\right)_{2}\right]_{4}\right\}^{3-}$. J. Am. Chem. Soc. 117, 681-691 (1995).

27. Taylor, M. S. \& Jacobsen, E. N. Asymmetric catalysis by chiral hydrogen-bond donors. Angew. Chem. Int. Ed. 45, 1520-1543 (2006).

28. Melchiorre, P., Marigo, M., Carlone, A. \& Bartoli, G. Asymmetric aminocatalysis-gold rush in organicchemistry. Angew. Chem. Int. Ed. 47, 6138-6171 (2008).

29. Couck, S. et al. An amine-functionalized MIL-53 metal - organic framework with large separation power for $\mathrm{CO}_{2}$ and $\mathrm{CH}_{4}$. J. Am. Chem. Soc. 131, 6326-6327 (2009).

30. Vaidhyanathan, R. Direct observation and quantification of $\mathrm{CO}_{2}$ binding within an amine-functionalized nanoporous solid. Science 330, 650-653 (2010). 
31. Pham, T. et al. Understanding hydrogen sorption in a metal - organic framework with open-metal sites and amide functional groups. J. Phys. Chem C117, 9340-9354 (2013).

32. Chisholm, M. H. \& Zhou, Z. P. Concerning the mechanism of the ring opening of propylene oxide in the copolymerization of propylene oxide and carbon dioxide to give poly(propylene carbonate). J. Am. Chem. Soc. 126, 11030-11039 (2004).

33. Ishimoto, R., Kamata, K. \& Mizuno, N. A. Highly active protonated tetranuclear peroxotungstate for oxidation with hydrogen peroxide. Angew. Chem. Int. Ed. 51, 4662-4665 (2012).

34. Aggrawal, V. K., Lopin, C. \& Dandrinelli, F. New insights in the mechanism of amine catalyzed epoxidation: dual role of protonated ammonium salts as both phase transfer catalysts and activators of oxone. J. Am. Chem. Soc. 125, 7596-7601 (2003).

35. Yoshizawa, M., Tamura, M. \& Fujita, M. Diels-alder in aqueous molecular hosts: unusual regioselectivity and efficient catalysis. Science 312, 251-254 (2006).

36. Liao, P. Q. et al. Strong and dynamic $\mathrm{CO}_{2}$ sorption in a flexible porous framework possessing guest chelating claws. J. Am. Chem. Soc. 134, 17380-17383 (2012).

37. Gassensmith, J. J. et al. Strong and reversible binding of carbon dioxide in a green metal-organic framework. J. Am. Chem. Soc. 133, 15312-15315 (2011).

38. Srikanth, C. S. \& Chuang, S. S. C. Spectroscopic investigation into oxidative degradation of silica-supported amine sorbents for $\mathrm{CO}_{2}$ capture. Chem. Sus. Chem. 5, 1435-1442 (2012).

39. McDonald, T. M. et al. Capture of carbon dioxide from air and flue gas in the alkylamine-appended metal-organic framework mmen- $\mathrm{Mg}_{2}$ (dobpdc). J. Am. Chem. Soc. 134, 7056-7065 (2012).

40. Nijem, N. et al. Understanding the preferential adsorption of $\mathrm{CO}_{2}$ over $\mathrm{N}_{2}$ in a flexible metal-organic framework. J. Am. Chem. Soc. 133, 12849-12857 (2011).

41. Luo, S. Z. et al. Functionalized chiral ionic liquids as highly efficient asymmetric organocatalysts for Michael addition to nitroolefins. Angew. Chem. Int. Ed. 45, 3093-3097 (2006)

42. Katritzky, A. R. et al. Preparation of nitropyridines by nitration of pyridines with nitric acid. Org. Biomol. Chem. 3, 538-541 (2005).

43. Ginsberg, A. P. Inorganic syntheses, Copyright (C) 1990, By. Inorg. Synth., Inc 27, 81-82 (1990).

44. SMART, Data collection software (version 5.629) (Bruker AXS Inc., Madison, WI, 2003).

45. SAINT, Data reduction software (version 6.45) (Bruker AXS Inc., Madison, WI, 2003).
46. Sheldrick, G. M. SHELXTL97, Program for Crystal Structure Solution (University of Göttingen: Göttingen, Germany, 1997).

\section{Acknowledgements}

This study was supported by the National Natural Science Foundation of China (21421005, 21231003 and U1304201), the Basic Research Program of China (2013CB733700), and the Program for Changjiang Scholars and Innovative Research Team in University (IRT1213).

\section{Author contributions}

Q.X.H., C.H. and C.Y.D. conceived and designed the experiments. Q.X.H., B.Q. and W.M.R. performed the experiments. C.H., J.Y.N. and C.Y.D. contributed materials and analysis tools. Q.X.H., C.H. and C.Y.D. co-wrote the paper. All authors discussed the results and commented on the manuscript.

\section{Additional information}

Accession codes: The X-ray crystallographic coordinates for structures reported in this study have been deposited at the Cambridge Crystallographic Data Centre (CCDC), under deposition numbers 1063826-1063827. These data can be obtained free of charge from The Cambridge Crystallographic Data Centre via www.ccdc.cam.ac.uk/data_request/cif.

Supplementary Information accompanies this paper at http://www.nature.com/ naturecommunications

Competing financial interests: The authors declare no competing financial interests.

Reprints and permission information is available online at http://npg.nature.com/ reprintsandpermissions/

How to cite this article: Han, Q. et al. Polyoxometalate-based homochiral metal-organic frameworks for tandem asymmetric transformation of cyclic carbonates from olefins. Nat. Commun. 6:10007 doi: 10.1038/ncomms10007 (2015).

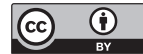

This work is licensed under a Creative Commons Attribution 4.0 International License. The images or other third party material in this article are included in the article's Creative Commons license, unless indicated otherwise in the credit line; if the material is not included under the Creative Commons license, users will need to obtain permission from the license holder to reproduce the material. To view a copy of this license, visit http://creativecommons.org/licenses/by/4.0/ 